\title{
La protection des droits fondamentaux dans l'Union européenne
}

\section{Retour vers le futur de l'avis $\mathbf{2 / 1 3}$ de la Cour de justice, de l'adhésion de l'UE à la CEDH et de l'Union européenne elle-même}

\section{The protection of fundamental rights in the European Union
Opinion 2/13 of the Court of Justice and the EU accession to the ECHR: Back to the Future

Karine Caunes ${ }^{1}$

(C) ERA 2016

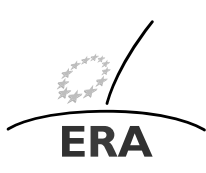

EUROPÄISCHE RECHTSAKADEMIE ACADEMY OF EUROPEAN LAW ACADEMIE DE DROIT EUROPEEN ACCADEMIA DI DIRITTO EUROPEO TRIER - TREVES - TREVIRI

Mots clés Droits fondamentaux · Adhésion · Souveraineté · Primauté

Keywords Fundamental rights · Accession · Sovereignty · Primacy

La protection des droits fondamentaux est au cœur de la construction européenne et de la légitimité, voire des tentatives de (dé)légitimation de l'Union européenne. Elle est également au cœur des activités de l'ERA et de sa Section Droit Public, lesquelles ont été marquées en 2015 notamment par l'analyse de la portée et des conséquences de l'avis $2 / 13$ du 18 décembre 2014 de la Cour de justice de l'Union européenne relatif à l'adhésion de l'Union européenne (UE) à la Convention européenne des droits de l'homme (CEDH). Beaucoup d'encre a coulé relativement à cette affaire et, alors que la Commission européenne continue de plancher sur le sujet, inscrit à l'ordre du jour de l'agenda du Conseil «Justice et affaires intérieures » des 8 et 9 juin prochains, reste pour les européanistes et professionnels du droit une question fondamentale relative à l'adhésion mais également plus gravement peut-être au projet européen lui-même : quo vadis?

Une intime compréhension de l'enjeu de cette question repose sur une analyse du raisonnement suivi par la Cour de justice dans l'avis 2/13 et de la portée de cet avis. Il convient de distinguer en effet parmi les arguments utilisés par la Cour ceux qui affèrent à certaines caractéristiques de l'Union européenne soulevant légitimement la

Les opinions exprimées dans cet éditorial sont celles de l'auteur uniquement et ne reflètent en aucune façon la position officielle de l'Académie de Droit Européen (ERA).

Dr. K. Caunes

kcaunes@era.int

1 Section Droit Public, Académie de droit européen (ERA), Metzer Allee 4, 54295 Trèves, Allemagne 
question de la compatibilité du projet d'accord relatif à l'adhésion de l'UE à la CEDH avec les traités UE et FUE, de ceux qui affèrent à l'autonomie du droit de l'Union, beaucoup plus problématiques et objets de la présente analyse. Pour paraphraser le fameux arrêt Costa c. ENEL rendu 50 ans plus tôt, le 15 juillet 1964, dans l'affaire numéro 6/64, si la question de l'adhésion devait se représenter devant la Cour, celle-ci ne pourrait s'obstiner à suivre cette ligne argumentative sans que l'ordre juridique dont elle est la gardienne ne perde son caractère d'Union et sans que soit mise en cause la base juridique de l'Union elle-même. L'avis 2/13 soulève en effet deux questions fondamentales : 1/ Entre droits fondamentaux et souveraineté, quel est le moyen et quelle est la fin ? 2/ Intégrité et intégration sont-elles compatibles ? Répondre à ces questions suppose d'adopter une méthodologie chère à la Cour dans le domaine par exemple des compétences de l'Union européenne : l'analyse du parallélisme entre le plan interne et le plan externe. Quant à la dimension intra-européenne : l'intégrité des ordres juridiques des Etats membres est-elle soluble dans l'intégration européenne? Quant à la dimension externe : l'intégrité de l'ordre juridique de l'Union européenne est-elle soluble dans son intégration sur le plan international?

Commençons par le plan interne. Le lien entre droits fondamentaux (des citoyens) et souveraineté (des citoyens dans une démocratie) explique que la protection des droits fondamentaux ait toujours été intimement liée à l'intégration européenne par le droit et se trouve au cœur des rapports entre Union européenne et Etats membres. Comme l'illustre parmi tant d'autres exemples la saga So lange, l'acceptation du principe de primauté du droit de l'Union européenne sur le droit national par les juridictions nationales a été conditionnée par la reconnaissance et le développement par la Cour de justice de son rôle de juge des droits fondamentaux en matière européenne. Si la Cour de justice reconnaissait dès 1969 la possible consécration de droits fondamentaux au rang constitutionnel de principes généraux du droit, c'est dans son dialogue avec les juridictions nationales et notamment autour de la question de la primauté du droit de l'Union européenne et de ses éventuelles limites qu'elle a développé une véritable doctrine de protection des droits fondamentaux dans le contrôle de la validité des normes juridiques européennes et de leur interprétation. Nombre de juridictions nationales appose encore d'ailleurs comme limite à leur acceptation du principe de primauté la protection, par leurs soins et sur le fondement de leur constitution, des droits fondamentaux. C'est dans ce contexte que s'est développée l'intégration européenne par le biais de la mise en place d'un système de protection équivalente des droits fondamentaux entre l'Union européenne et les Etats membres, permettant à chaque ordre juridique impliqué de préserver sa souveraineté. Ce système de protection équivalente des droits fondamentaux repose sur l'idée que chacun des ordres juridiques impliqués ou intégrés affirme le droit souverain d'assurer la protection ultime des droits ou normes fondamentales en son royaume. Ce faisant, les juges, européens ou nationaux, gardiens de leur ordre juridique, assurent non seulement la bonne application des normes juridiques pertinentes mais encore la sauvegarde de l'intégrité de leur ordre juridique, en d'autres termes, sa souveraineté. Et l'on pourrait se demander de surcroît, sur le plan de la philosophie politique, si un tel pluralisme, un tel système de poids et de contrepoids en matière de protection des droits fondamentaux, n'est pas le propre d'un système politique démocratique voire affirmer, à l'instar d'Hanna Arendt dans La condition de l'homme moderne, que le pluralisme est la condition de la démocratie. 
Ainsi, à la question : "l'intégrité des ordres juridiques des Etats membres estelle soluble dans l'intégration européenne? », la réponse est tout simplement non. Ou peut-être pourrions-nous dire que la question est tout simplement mal formulée car reposant sur des modèles de la relation Union européenne / Etats membres selon lesquels la primauté, et donc la souveraineté sur le plan formel, de l'une et des autres sont mutuellement exclusives. Or, sur le plan matériel, si la devise de l'Union européenne est « Unis dans la diversité », c'est avant tout parce qu'elle illustre un type de relations entre systèmes juridiques préservant l'intégrité des ordres juridiques parties, lesquels en constituent la richesse. Une intégrité européenne et nationale non pas protectionniste et recroquevillée sur elle-même, mais protectrice de ses valeurs et donc par essence ouverte vis-à-vis de l'autre, cet autre traité comme un autre soi-même pour retourner l'heureuse formule de Paul Ricœur mais dont l'identité alter n'est pas soluble dans la sienne propre. En somme, une telle intégrité ouverte repose sur une identité-ipséité qui prend le changement et donc l'adaptation à celui-ci comme une de ses caractéristiques intrinsèques et vitales, à l'opposé d'une intégrité fermée reposant sur une identité-mêmeté nourrie de l'illusion de la permanence, incapable de s'adapter au changement forcément extérieur et forcément conçu comme une menace. Cette dernière conception de l'intégrité, qui semble s'être funestement faufilée dans la trame de l'avis 2/13 de la Cour de justice, enferme la souveraineté dans un solipsisme illustrant la devise de Highlander, ô combien inadaptée à la nature même de l'Union européenne mais également des relations internationales : «il ne peut en rester qu'un ».

Par ailleurs, toujours sur le plan interne, à la question : «entre droits fondamentaux et souveraineté : quel est le moyen et quelle est la fin ? », la réponse ne peut que se trouver dans un cercle vertueux dans lequel la souveraineté appartient aux peuples qui l'exercent et la protègent par le biais de leurs droits fondamentaux. D'un côté, les droits fondamentaux sont l'instrument de la protection de la souveraineté des peuples européens et donc de la démocratie et de leur liberté. De l'autre, la souveraineté, dans un système démocratique, est l'instrument de la protection des droits fondamentaux des citoyens, en l'occurrence européens. Dans les deux cas et dans une perspective kantienne, l'Homme est toujours la fin. Ce cercle vertueux ou système de balancier entre droits fondamentaux, au cœur de toute constitution matérielle d'un système politique démocratique, et souveraineté, incarnée sur le plan formel par la norme d'habilitation ultime du système juridique considéré, est de la fabrique même de la construction européenne qu'illustre le système de protection équivalente des droits fondamentaux. On s'aperçoit alors que l'émergence de la question précédemment formulée : «entre droits fondamentaux et souveraineté : quel est le moyen et quelle est la fin? » est le signe d'un malaise dans le projet de civilisation européenne. Si souveraineté et droits fondamentaux se trouvent dissociés voire opposés, cela signifie qu'ils ne remplissent plus forcément leur mission démocratique. Or, ce sont de tels doutes qui semblent avoir saisi la gens européenne à la lecture de l'avis 2/13 de la Cour de justice et, si un certain malaise perdure, c'est en raison des implications de cet avis quant à la nature du projet européen et à son futur. Passons donc à l'analyse du volet externe de la jurisprudence de la Cour.

Si la Cour de justice a légitimé son action sur le plan interne et l'existence même de l'Union européenne en devenant championne de la protection des droits fondamentaux en son royaume, d'aucuns pourraient alors trouver paradoxale qu'elle ne se 
considère pas pour autant comme une Cour des droits de l'homme. C'est là oublier la dimension externe de son action avec en toile de fond le cercle vertueux, brisé ?, entre protection des droits fondamentaux et souveraineté.

Si la Cour a pu s'ériger en championne de la défense des droits fondamentaux sur le plan international dans l'affaire Kadi, c'est en limitant la portée du principe de primauté de la Charte des Nations Unies, en particulier des obligations relatives à la mise en œuvre des résolutions du Conseil de sécurité adoptées au titre du chapitre VII de cette Charte, par l'exclusion du champ de ce principe du droit primaire européen et, en particulier, des principes généraux dont font partie les droits fondamentaux. Si d'aucuns ont pu voir ici de la part de la Cour une volonté de préservation de la souveraineté ou de l'autonomie de l'ordre juridique européen, celle-ci a pu être justifiée pour certains par l'alliance vertueuse entre protection de la souveraineté européenne et protection des droits fondamentaux, illustrant la mise en place sinon d'un embryon de système de protection équivalente sur le plan international, du moins la préservation d'une forme d'état de droit, internationale. Un tel raisonnement s'avère difficilement soutenable dans le cas de l'avis $2 / 13$.

Afin de justifier son avis négatif relatif à l'adhésion de l'Union européenne à la Convention européenne des droits de l'homme, la Cour s'est fondée notamment sur l'atteinte susceptible d'être portée aux caractéristiques spécifiques du droit de l'Union et à l'autonomie de ce dernier. Si la préservation des caractéristiques spécifiques du droit de l'Union est bien posée en condition de l'adhésion de l'Union par le Protocole $\mathrm{n}^{\circ} 8$ relatif à l'article 6 , paragraphe 2 , du traité sur l'Union européenne sur l'adhésion de l'Union à la convention européenne de sauvegarde des droits de l'homme et des libertés fondamentales, force est cependant de constater que tel n'est pas le cas de «l'autonomie du droit de l'Union européenne ». Or, ce qui paraît problématique dans l'avis de la Cour est la conception de l'autonomie, autrement dit de la souveraineté adoptée par celle-ci. L'association particulière entre «caractéristiques spécifiques » et «autonomie » opérée par la Cour est le signe d'une conception figée et protectionniste de l'identité de l'Union européenne, une identité-mêmeté nourrit de l'illusion de la permanence, incapable de s'adapter au changement forcément extérieur et forcément conçu comme une menace, allant à l'encontre de la nature même de l'Union européenne comme expliqué précédemment.

En fondant son raisonnement sur la préservation nécessaire de l'«autonomie » européenne, la Cour méconnaît la nature même du concept d'autonomie ou souveraineté et tombe dans l'écueil de son paradoxe. En effet, le paradoxe de la souveraineté consiste à trouver la réponse à la question suivante : la souveraineté peut-elle être limitée ? Comment un ordre juridique souverain peut-il se soumettre à des obligations externes ? Réponse : en étant la source de sa propre soumission, c'est-à-dire en y ayant consenti. Souveraineté-prérogative (choix) et souveraineté-obligation (soumission) sont les deux facettes d'une même pièce. Toute expression de souveraineté par la conclusion d'un traité international est également une limitation de celle-ci. La souveraineté apparaît alors comme un concept opérationnel en ce qu'elle ne fait pas obstacle aux relations avec d'autres systèmes juridiques tout en permettant de préserver les caractéristiques essentielles inhérentes audit système. La souveraineté ainsi conçue est le concept par excellence de l'identité-ipséité, une identité ouverte.

Or, en l'espèce, le choix souverain d'adhérer à la Convention européenne des droits de l'homme a été librement consenti par l'Union européenne puisqu'il se 
trouve consacré à l'article 6 paragraphe 2 du traité sur l'Union européenne. Ainsi il ne revient pas à la Cour de justice de contrôler la validité de ce choix lui-même, à valeur constitutionnelle, mais simplement les modalités juridiques de mise en œuvre de ce choix. Celles-ci ne pourraient être remises en cause par la Cour que si elles avaient pour conséquence de porter atteinte aux diverses conditions d'adhésion, à valeur constitutionnelle elles aussi, rappelées par la Cour de justice aux paragraphes 160 à 162 de son avis et décrites au paragraphe 164 comme «les caractéristiques spécifiques de l'Union et de son droit ». Il convient de noter que la Cour inclut dans celles-ci des caractéristiques issues de sa jurisprudence c'est-à-dire :

«les caractéristiques spécifiques tenant à la nature même du droit de l'Union. En particulier, comme la Cour l'a relevé à maintes reprises, le droit de l'Union se caractérise par le fait d'être issu d'une source autonome, constituée par les traités, par sa primauté par rapport aux droits des Etats membres ainsi que par l'effet direct de toute une série de dispositions applicables à leurs ressortissants et à eux-mêmes » (paragraphe 166 de l'avis 2/13)

De plus,

«l'autonomie dont jouit le droit de l'Union par rapport aux droits des États membres ainsi que par rapport au droit international impose que l'interprétation de ces droits fondamentaux soit assurée dans le cadre de la structure et des objectifs de l'Union » (paragraphe 170 de l'avis 2/13)

Enfin,

«Pour garantir la préservation des caractéristiques spécifiques et de l'autonomie de cet ordre juridique, les traités ont institué un système juridictionnel destiné à assurer la cohérence et l'unité dans l'interprétation du droit de l'Union » (paragraphe 174 de l'avis 2/13)

Quelques remarques s'imposent. Premièrement, le mandat de la Cour est limité au contrôle du respect des conditions d'adhésion à valeur constitutionnelle sans quoi celle-ci agirait ultra vires. Deuxièmement, la ligne semble être ténue entre contrôle du choix souverain de l'Union d'adhérer à la CEDH consacré à l'article 6 paragraphe 2 TUE, lequel n'est pas de la compétence de la Cour, et contrôle de la préservation de l'autonomie du droit de l'Union, condition d'adhésion selon la Cour, de son ressort. Troisièmement, le lien opéré entre autonomie du droit de l'Union et compétence exclusive d'interprétation de la Cour met celle-ci dans la délicate situation en l'espèce d'être pour ainsi dire juge et partie soulevant la question de principe de l'étendue de son contrôle. Or, quelques doutes surgissent de par l'usage que fait la Cour d'une conception fermée de l'autonomie au stade de l'adhésion à la CEDH.

En effet, il apparaît à la lecture de l'avis 2/13 de la Cour que la caractéristique essentielle de cette autonomie ou la préservation de celle-ci consiste en la compétence exclusive de la Cour à interpréter in fine toute question pouvant toucher, de près ou de loin ou simplement hypothétiquement le droit de l'Union européenne, et même dans des domaines telle la PESC où elle n'est elle-même pas compétente de principe. Il est cependant difficile de comprendre la raison pour laquelle la Cour brandit l'argument de la préservation de l'autonomie de l'ordre juridique européen et de sa compétence exclusive d'interprétation au stade de l'adhésion à la CEDH. 
Tout d'abord, pourquoi la Cour ne pourrait-elle pas, après l'adhésion, se fonder sur les valeurs communes de l'Union européenne, noyau constitutionnel de l'Union consacré à l'article 2 TUE, dont la valeur juridique supplante celle du consentement à l'adhésion à la $\mathrm{CEDH}$ et le respect prime sur le respect des obligations internationales y attenantes, afin d'adopter par exemple une position différente de celle de la Cour européenne des droits de l'homme dans un cas concret? N'est-ce pas somme toute un raisonnement similaire que la Cour a fait prévaloir dans l'affaire Kadi ? Si la Cour ne préservait pas ces valeurs, l'Union européenne perdrait en effet son autonomie. Il s'agirait d'un cas dans lequel l'Union ne pourrait remplir ses obligations internationales sans que l'ordre juridique dont la Cour est la gardienne ne perde son caractère d'Union et sans que soit mise en cause la base juridique de l'Union ellemême pour paraphraser de nouveau l'arrêt Costa c. ENEL. Cependant, il n'y a pas de risque que l'Union perde son autonomie en deçà de ce stade et la Cour dispose des outils juridiques nécessaires à la préservation de l'autonomie du droit de l'Union après l'adhésion.

Ensuite, en cas d'adhésion à la $\mathrm{CEDH}$, la seule conséquence du non-respect d'un arrêt de la Cour européenne des droits de l'homme par exemple serait la même que celle encourue par les Etats membres eux aussi parties à la CEDH dans une situation similaire : la mise en jeu classique de la responsabilité internationale, ici de l'Union européenne, par le jeu d'un règlement politique de l'inexécution de l'arrêt de la Cour européenne des droits de l'homme décidé par le Comité des Ministres. A la différence de l'Union européenne, le Conseil de l'Europe reste un système juridique de type international et les arrêts de la Cour ne sont dotés que d'un effet déclaratoire. Le Conseil de l'Europe et l'Union européenne restant deux systèmes juridiques bien distincts, en aucun cas la compétence exclusive d'interprétation des traités UE et FUE de la Cour de justice ne pourrait être remise en cause.

Enfin, si la Cour de justice devait abonder dans le sens de la Cour européenne des droits de l'homme sur une question donnée, ce serait évidemment afin de respecter les engagements internationaux pris par l'Union mais ce serait de manière première sur le fondement du droit de l'Union et non de la CEDH.

Ainsi, l'adhésion de l'Union européenne à la CEDH ne peut avoir pour conséquence de porter atteinte à la compétence exclusive d'interprétation des traités UE et FUE de la Cour de justice, celle-ci ayant les instruments nécessaires à la préservation de l'autonomie du droit de l'Union après l'adhésion. De plus, cette dernière permettrait de développer une forme de système de protection équivalente des droits fondamentaux propre à la relation entre Union européenne et Conseil de l'Europe, propice au développement de l'état de droit européen et international et à laquelle la doctrine Bosphorus de présomption d'équivalence de protection des droits fondamentaux entre les deux systèmes pourrait servir d'embryon. Par sa position défensive, la Cour, au lieu de préserver l'autonomie de l'ordre juridique européen, contribue à l'affaiblir. En l'espèce, le cercle vertueux entre droits fondamentaux et souveraineté est brisé puisque protection de la souveraineté européenne et protection accrue des droits fondamentaux en Europe par l'adhésion de l'Union européenne à la Convention européenne des droits de l'homme sont mis dos à dos. Dans un tel duel, nul ne gagne et certainement pas la démocratie européenne. Est remis en cause non seulement l'état de droit international mais aussi l'état de droit européen, le modèle même 
d'alliance entre préservation de la souveraineté et protection des droits fondamentaux sur lequel repose l'Union, voire également par conséquent le fondement des principes de coopération loyale et de confiance mutuelle si chers à la Cour de justice. Comme noté par la Cour aux paragraphes 167 et 168 de l'avis 2/13:

«Ces caractéristiques essentielles du droit de l'Union ont donné lieu à un réseau structuré de principes, de règles et de relations juridiques mutuellement interdépendantes liant, réciproquement, l'Union elle-même et ses États membres, ainsi que ceux-ci entre eux, lesquels sont désormais engagés, comme il est rappelé à l'article ler, deuxième alinéa, TUE, dans un "processus créant une union sans cesse plus étroite entre les peuples de l'Europe».

Une telle construction juridique repose sur la prémisse fondamentale selon laquelle chaque État membre partage avec tous les autres États membres, et reconnaît que ceux-ci partagent avec lui, une série de valeurs communes sur lesquelles l'Union est fondée, comme il est précisé à l'article 2 TUE. Cette prémisse implique et justifie l'existence de la confiance mutuelle entre les États membres dans la reconnaissance de ces valeurs et, donc, dans le respect du droit de l'Union qui les met en æuvre ».

Ce faisant, est remise en cause également la viabilité de toute convention internationale sur l'autel de la souveraineté mal comprise de l'Union. Et l'on ne peut alors regarder rétrospectivement les autres positions de la Cour qu'avec un certain malaise ou un mal à l'âme européenne. Il ne paraît alors plus anodin que toujours le 18 décembre 2014, la Cour de justice ait refusé d'étendre les cas d'effet direct des dispositions du GATT de 1994 dans l'affaire C-306/13 dite Van Parys II. Il ne paraît hélas guère plus surprenant que la Cour ait sacrifié la possibilité d'une meilleure protection des droits des personnes handicapées, car faut-il le rappeler, l'Union européenne est déjà partie à un traité en matière de droits de l'homme : la Convention des Nations Unies relative aux droits des personnes handicapées, sur l'autel de la sacro-sainte autonomie du droit de l'Union européenne, en privant d'effet direct ladite Convention par un arrêt du 18 mars 2014 dans l'affaire C-363/12 dite Z. Selon cette grille de lecture, il semble que la Cour ait développé une vision exclusive de la souveraineté sur le plan international en adoptant une stratégie visant à bloquer toute concurrence possible soit en bloquant la ratification de traités internationaux, soit s'ils ont déjà été ratifiés en les privant d'effet direct dans l'ordre juridique européen ou en limitant la portée du principe de primauté du droit international comme dans l'affaire Kadi.

Il est à espérer cependant qu'il ne s'agit en réalité pas de la stratégie de la Cour mais uniquement d'une impression erronée que pourrait donner l'avis 2/13. La Cour n'a certes pas cherché par cet avis à remettre en cause l'état de droit international ni encore moins l'état de droit européen. En effet, pour finir le parallélisme entre plan interne et externe, en ce qui concerne la question : (entre droits fondamentaux et souveraineté, quel est le moyen et quelle est la fin ?) la Cour donnerait l'impression par l'avis 2/13 que les droits fondamentaux ne sont qu'un instrument visant à la sauvegarde de la souveraineté de l'Union européenne ce qui pourrait expliquer l'assertion selon laquelle la Cour de justice n'est pas une cour des droits de l'homme mais aurait le désavantage de contribuer à la délégitimation du projet européen car enfin quelle 
serait la valeur d'une souveraineté acquise au détriment des valeurs fondatrices de l'UE de respect des droits de l'homme et de démocratie?

A la question : (l'intégrité de l'ordre juridique de l'Union européenne est-elle soluble dans son intégration sur le plan international ?) par sa position protectionniste dans l'avis 2/13, la réponse de la Cour semble être que oui mais il est à espérer que le génie de la Cour s'exprimera prochainement de nouveau lorsque les obstacles relatifs uniquement aux caractéristiques spécifiques de l'Union seront levés et qu'elle fournira le pendant de l'arrêt Costa c. ENEL sur le plan international, contribuant ainsi à asseoir la reconnaissance de l'Union européenne en tant qu'acteur de droit international.

Plus généralement, à l'heure où les crises économiques ou migratoires sévissent, l'Union européenne a d'autant plus besoin que la Cour assure son rôle de gardienne de l'ordre juridique européen et de ses valeurs. Il convient également de souligner qu'elle a par ailleurs adopté dans le domaine de la protection des données par exemple des positions courageuses visant à la sauvegarde des droits des individus que ce soit à travers l'invalidation de la directive sur la détention des données dans son arrêt du 8 avril 2014 dans les affaires jointes C-293/12 et C-594/12 Digital Rights Ireland et Seitlinger ou bien de l'accord Safe Harbour relatif au transfert des données personnelles de l'Europe vers les Etats-Unis dans son arrêt du 6 octobre 2015 dans l'affaire C-362/14 Schrems. A n'en point douter, c'est avec le même courage et la même sagacité que la Cour de justice saura, après l'adhésion de l'Union européenne à la CEDH, défendre les valeurs communes de l'Union européenne et, par le biais d'un dialogue avec la Cour européenne des droits de l'homme et la création d'un véritable système inédit de protection équivalente des droits fondamentaux, faire rayonner ce modèle sur le continent européen. 